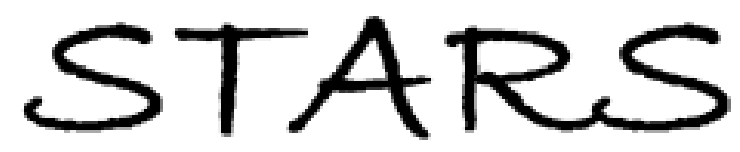

University of Central Florida

STARS

Faculty Bibliography 2000s

Faculty Bibliography

$1-1-2003$

\title{
Coherence properties near interfaces of random media
}

\author{
A. Apostol \\ University of Central Florida
}

A. Dogariu

University of Central Florida

Find similar works at: https://stars.library.ucf.edu/facultybib2000

University of Central Florida Libraries http://library.ucf.edu

This Article is brought to you for free and open access by the Faculty Bibliography at STARS. It has been accepted for inclusion in Faculty Bibliography 2000s by an authorized administrator of STARS. For more information, please contact STARS@ucf.edu.

\section{Recommended Citation}

Apostol, A. and Dogariu, A., "Coherence properties near interfaces of random media" (2003). Faculty Bibliography 2000s. 3596.

https://stars.library.ucf.edu/facultybib2000/3596

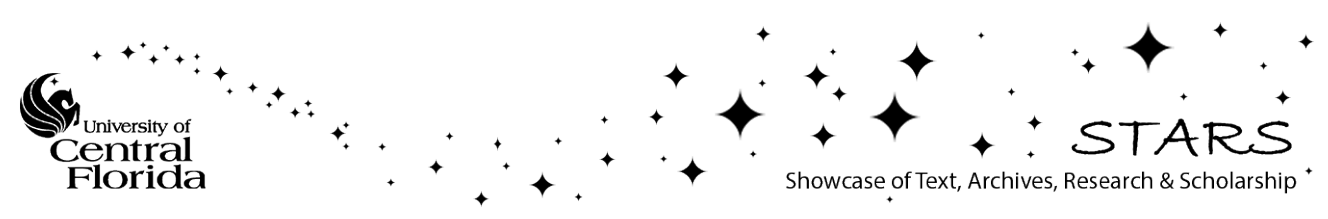




\title{
Coherence properties near interfaces of random media
}

\author{
A. Apostol and A. Dogariu \\ School of Optics/CREOL University of Central Florida, Orlando, Florida 32816
}

(Received 12 February 2003; published 30 May 2003)

\begin{abstract}
The spatial coherence theory of optical fields radiated by statistically homogeneous, infinite, planar sources does not account for detailed surface characteristics. However, coherence properties change with the angular distribution of the intensity radiated from such a source, which, in turn, depends on the physical properties of the sample. We show that second-order correlations of the optical field measured at several wavelengths away from the surface of a highly inhomogeneous medium relate to the statistical characteristics of the interface.
\end{abstract}

DOI: 10.1103/PhysRevE.67.055601

PACS number(s): 42.25.Kb, 42.50.Ar, 07.79.Fc

A highly inhomogeneous medium is known to produce significant field fluctuations and a radiant intensity that is regarded as typical for incoherent sources. It was a long-time belief that multiple scattering washes out all the structural information and that such a random medium would behave as a perfect Lambertian diffuser. The optical coherence theory predicts that the quasihomogeneous Lambertian sources of radiation are not actually completely spatially incoherent; at a given optical frequency $\omega$, the field correlates over regions whose spatial dimensions are of the order of the wavelength $\lambda=2 \pi c / \omega$ [1]. In addition, the field coherence length does not depend on the distance $z$ from the interface when this distance is larger than the wavelength. One has to realize that this description is pertinent to coherence phenomena of radiation produced by a practically infinite source and does not account for detailed surface characteristics [1]. Note also that classical measurements of spatial coherence properties are usually performed at much larger distances $z$, where the limited size of the source cannot be ignored. In this case, the size of the coherence area is actually determined by the extent of the source as described by the Van Cittert-Zernike theorem [2].

The real scattering media on the other hand have rough interfaces that may affect the field correlation in the neighborhood of the surface. As a result, the statistical characteristics of the interface are expected to also influence the spatial coherence properties of the radiation emitted. In other words, the field coherence properties of the radiation originating from a bounded random medium should be related to the statistical characteristics of its interface.

Here we show how, using improved field-scanning techniques, the spatial coherence properties can be related to the optical characteristics of the surface. We report theoretical and experimental results demonstrating that the second-order spatial coherence of the field in the vicinity of a random medium depends on the interface statistical properties. Our results refer to physical situations where media with strong volume randomness are bounded by relatively smooth surfaces with slowly varying interfaces (surface roughness smaller than $\lambda / 2$ and surface correlation length larger than $3 \lambda / 2)$.

The angular dependence of light, which is diffusely transmitted through a random volume-scattering medium (VSM) with absorbing boundaries, is well described by the diffusion theory and it is known to have a cosine distribution [3].
However, real scattering media are bounded by interfaces that manifest refractive index mismatch and, moreover, are rough on the scale of the wavelength. As a result, the boundary conditions for the photon diffusion are modified and the angular distribution of intensity $J(\theta)$ changes. The effect of a real surface can be accounted for by considering an "effective" planar interface that radiates an intensity with an angular distribution different from the classical cosine dependence.

Because the angular distribution of radiant intensity can be directly related to the spatial coherence properties at the surface of two-dimensional sources of radiation [1], a description of the coherence properties of the radiation emitted by real media, i.e., bounded VSM can be developed, which considers the surface of the random medium as being an homogeneous, planar, statistically stationary source of optical radiation. Such a source, located in the $z=0$ plane, is characterized by a cross-spectral density function [4]

$$
W^{(0)}\left(\boldsymbol{\rho}_{1}, \boldsymbol{\rho}_{2}, \omega\right)=F^{(0)}\left(\boldsymbol{\rho}_{2}-\boldsymbol{\rho}_{1}, \omega\right)
$$

where $\boldsymbol{\rho}_{1}, \boldsymbol{\rho}_{2}$ are two-dimensional position vectors in the plane of the source and $\omega$ is the frequency of radiation. The field cross-spectral density function generated by the source in the half-space $z>0$ is then given by [4]

$$
\begin{aligned}
W\left(\mathbf{r}_{1}, \mathbf{r}_{2}, \omega\right)= & k^{2} \int_{-\infty}^{\infty} \int \widetilde{F}\left(k s_{x}, k s_{y}, \omega\right) \\
& \times \exp \left[i k\left(\mathbf{s r}_{1}-\mathbf{s}^{*} \mathbf{r}_{2}\right)\right] d s_{x} d s_{y}
\end{aligned}
$$

where $\widetilde{F}\left(k s_{x}, k s_{y}, \omega\right)$ is the two-dimensional Fourier transform of $F^{(0)}\left(\boldsymbol{\rho}_{1}-\boldsymbol{\rho}_{2}, \omega\right), \mathbf{s}$ is the unit vector direction, $\mathbf{k}$ $=k \mathbf{s}$, and $k=\omega / c$. Because we are concerned with fields that propagate few wavelengths away from the source, we neglect the contribution of high-frequency components and the cross-spectral density function of the field in a $z>0$ plane can be expressed as

$$
\begin{aligned}
W(\Delta x, \Delta y, \omega)= & k^{2} \int_{s_{x}^{2}+s_{y}^{2} \leqslant 1} \widetilde{F}\left(k s_{x}, k s_{y}, \omega\right) \\
& \times \exp \left[i k\left(s_{x} \Delta x+s_{y} \Delta y\right)\right] d s_{x} d s_{y},
\end{aligned}
$$


where $\mathbf{r}_{2}-\mathbf{r}_{1}=(\Delta x, \Delta y, 0)$ and $\mathbf{s}=\left(s_{x}, s_{y}, \sqrt{1-s_{x}^{2}-s_{y}^{2}}\right)$.

The radiant intensity is directly proportional to the inverse Fourier transform of the cross-spectral density function in the plane of the source [1],

$$
J_{\omega}(\mathbf{s})=k^{2} A \widetilde{F}\left(k s_{x}, k s_{y}, \omega\right) \cos ^{2} \theta,
$$

where $A$ is the source area and $\theta$ is the angle that the vector $\mathbf{s}$ makes with the normal to the source.

It follows from Eqs. (3) and (4) after changing from Cartesian coordinates to polar ones, i.e., using $\Delta x=r \cos \varphi$, $\Delta y=r \sin \varphi$ and $s_{x}=\rho \cos \phi, s_{y}=\rho \sin \phi$, that

$$
\begin{aligned}
W(r, \omega)= & \frac{1}{A} \int_{0}^{2 \pi} \int_{0}^{1} \frac{J\left(\arccos \sqrt{1-\rho^{2}}\right)}{1-\rho^{2}} \\
& \times \exp [i k r \rho \cos (\varphi-\phi)] \rho d \rho d \phi .
\end{aligned}
$$

This analysis suggests a way to predict the field coherence length $\sigma_{\mu}$ when the interface statistics in known. Using Eq. (5), the cross-spectral density can be numerically estimated for different distributions of the radiant intensity. Moreover, $J(\theta)$ is related to statistical properties at the surface of the sample. In order to obtain the transmitted light distribution as a function of the scattering angle, one has to develop a statistical description of the surface. The statistical character of the surface can be simply described by the probability density function of the random inclination of the surface normal with respect to the $z$ axis (local slope). In many cases, one can also assume that the distribution of the local slopes is Gaussian with zero mean and has a standard deviation $\sigma_{s}$.

For highly inhomogeneous media, one can consider that unpolarized light is incident upon the interface from the dense medium and it has a cosine angular distribution. Knowing the probability density for the local slope and using Fresnel's coefficients, one finds that the radiant intensity $J(\theta)$ is given by [5]

$$
\begin{aligned}
J(\theta)= & \int_{-\pi / 2}^{-\pi / 2} S\left[\alpha-\arcsin \left(\frac{n_{2}}{n_{1}} \sin (\theta+\alpha)\right)\right] \\
& \times T\left[\arcsin \left(\frac{n_{2}}{n_{1}} \sin (\theta+\alpha)\right)\right] P(\alpha) d \alpha,
\end{aligned}
$$

where $P(\alpha)$ is the probability density function of the local slope, $n_{2}, n_{1}$ are the refraction indices defining the surface, $S(x)=\cos (x)$ represents the angular distribution of the incident intensity, and $T(x)$ is the depolarized transmittance calculated as the average of the TE and TM transmittances. The radiation with angles of incidence beyond the critical angle does not contribute to the outgoing intensity.

The simple model taking into consideration the statistical character of the surface and the reflective nature of the boundary can explain the change in the angular dependence of radiant intensity $J(\theta)$. The procedure allows for both local slope standard deviation and the refractive index contrast to be accounted for. In this way, the angular distribution of the emitted intensity is directly related to the statistical proper-

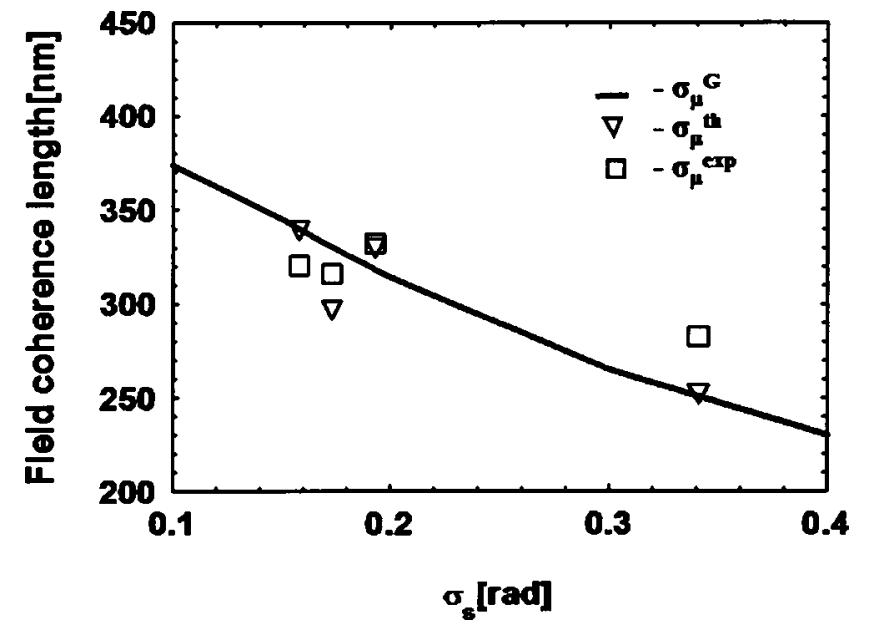

FIG. 1. Field correlation length as a function of the standard deviation $\sigma_{s}$ of local slope. Solid line: field coherence length $\sigma_{\mu}^{G}$ obtained using a Gaussian distribution with zero mean and standard deviation $\sigma_{s}$ for the local slope probability distribution function. Triangles: field coherence length $\sigma_{\mu}^{\text {th }}$ obtained using the experimental probability distribution function for the local slopes of the sample examined. Squares: coherence length $\sigma_{\mu}^{\exp }$ measured for the same samples. All calculations are for an index of refraction $n$ $=1.5$ and light wavelength $\lambda=488 \mathrm{~nm}$. The field correlation length is estimated from $1 / e$ values of the cross spectral density function $W(r, \omega)$.

ties of the real surface. This procedure was applied to VSM with different surface statistics.

In Fig. 1, the values of the field coherence length $\sigma_{\mu}$, defined as the 1/e values of the cross-spectral density, are plotted as a function of $\sigma_{s}$, the standard deviation of the local slope. The calculations presented are for $\lambda=488 \mathrm{~nm}$ and account just for the homogeneous components of the field. The continuous line in Fig. 1 represents the numerical evaluations of the field coherence length $\sigma_{\mu}^{G}$ using the procedure outlined above, when the local slope density probability distribution is considered to be a Gaussian with zero mean and standard deviation $\sigma_{s}$. One can see that $\sigma_{\mu}^{G}$ decreases with local slope standard deviation $\sigma_{s}$. Also, for large values of $\sigma_{s}$, when the radiant intensity $J(\theta)$ tends to a cosine angular distribution, the values of $\sigma_{\mu}^{G}$ decrease toward the coherence length for which $\sin \left(k \sigma_{\mu}\right) / k \sigma_{\mu}=1 / e$. This result can be easily understood: rough interfaces have the tendency to diminish the refractive index contrast, and therefore lead to a Lambertian source behavior corresponding to absorbing boundary conditions. The simple interface model together with the planar source concept explain how the field coherence properties near the source surface (at distances larger that the wavelength of light) depend on the statistical properties at the sample interface.

The theoretical predictions have also been examined experimentally. We measured the spatial coherence properties near the surface of various random, highly scattering media, that were illuminated in a transmission geometry. Here we report data collected on compact slabs made of calcium carbonate and kaolin microparticles with an average diameter of $400 \mathrm{~nm}$ and on slabs containing polystyrene spheres of 356 nm diameter. 
The experimental configuration is schematically presented in Fig. 2(a). A near-field scanning optical microscope (NSOM) was used to obtain atomic force microscope (AFM) topographical images as well as optical images at distances $z$ of 5-20 wavelengths from the interface. An $\mathrm{Ar}+$ ion laser $(\lambda=488 \mathrm{~nm})$ is used to illuminate the random medium and the transmitted field is coupled to the cantilevered near-field optical force sensor scanned along the sample. The scattered light is detected using a photomultiplier tube operating in the photon counting mode. The NSOM fiber probe with an aperture of $150 \mathrm{~nm}$ provides the possibility to scan both constant distance mode (for the AFM images) and constant height mode (for the optical images). Typical topographical and optical images are shown in Figs. 2(b) and 2(c). The rms heights of the surface are typically in the range of $\lambda / 4-\lambda / 2$. However, the correlation lengths of the scanned surfaces are larger than the wavelength of light, ranging from $3 \lambda / 2$ to $3 \lambda$.

The intensity distributions are measured by scanning across the sample at a constant distance $z$ and collecting images over a $5 \times 5 \mu \mathrm{m}$ area. The second-order intensity correlations are then evaluated and the field correlation function is calculated using standard properties of Gaussian random variables [2]. We evaluated numerically the field correlation functions and estimated the average radius of the cross section area where the field correlation decreases at 1/e of its maximum and consider the width of the field correlation as a measure of the field coherence length. Typical values of the experimental field coherence length $\sigma_{\mu}^{\exp }$ and of local slope standard deviation $\sigma_{s}$ are presented in Fig. 1. When estimating the field coherence lengths, the data were corrected with the point spread function of the probe.

A direct comparison between the experimental data and the theoretical values of the coherence length $\sigma_{\mu}^{\text {th }}$ is also presented in Fig. 1. The theoretical values $\sigma_{\mu}^{\text {th }}$ were calculated using the experimental values of the local slope probability density functions and Eq. (5). The local slopes and their standard deviation $\sigma_{s}$ were determined from the AFM topographical data and the heights were sampled with a lateral or vertical step that equals the size of the tip. Note that the values of the local slope probability density function used in the calculations labeled by triangles in Fig. 1 can be different from the Gaussian slope distribution with zero mean assumed in the theoretical results presented as a continuous curve in Fig. 1. A remarkable agreement is obtained with the simple model described here; the discrepancy between the theoretical and experimental values of the coherence length is less than $10 \%$. One can conclude that this concept of a planar source together with the model for the angular distribution of the radiant intensity qualitatively describe the second-order statistical properties of the field emitted close to the surface of a highly random medium. It also provides a direct relationship between the measurable statistical properties of the optical field at several wavelengths away from the surface and the statistical properties of an equivalent planar source of radiation which, in turn, are determined by the physical properties of the random medium.

The direct relationship between $\sigma_{\mu}$ and the topological characteristics of the surface can be used to identify the midscale surface inhomogeneities while a larger surface is
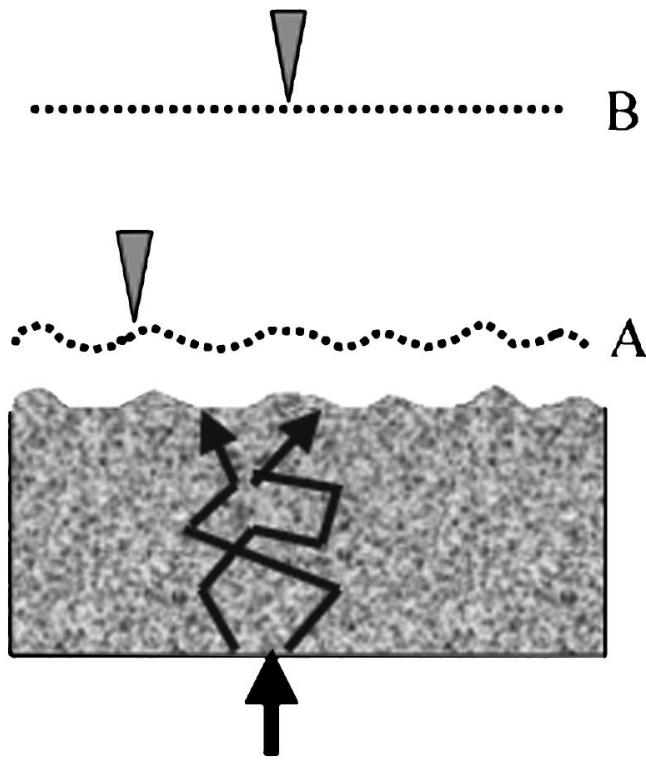

a

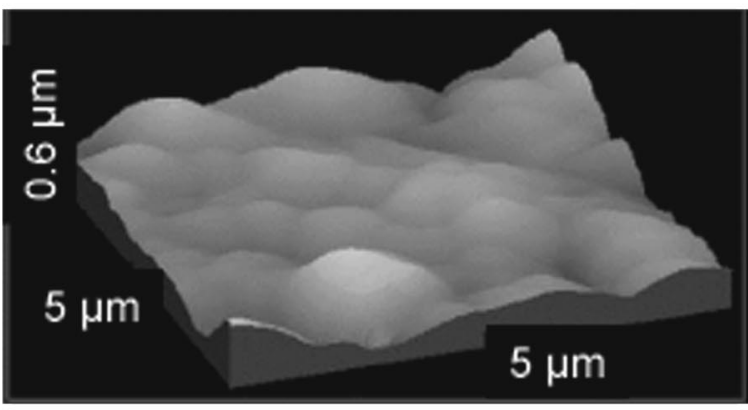

b

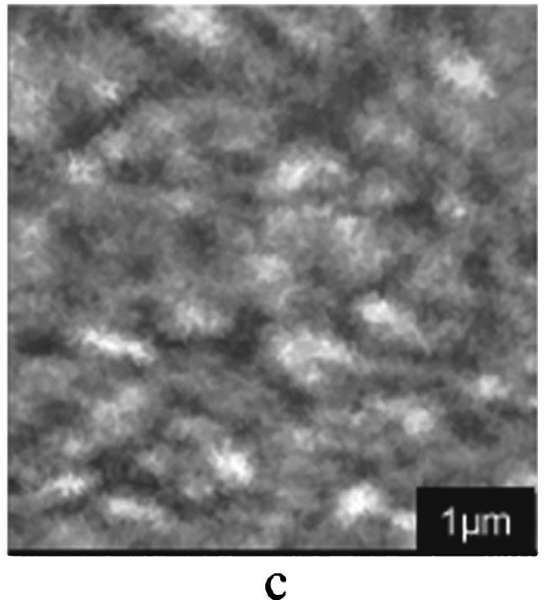

FIG. 2. (a) Experimental setup. A 150-nm-aperture probe was used to scan in both constant distance $(A)$ and constant height mode (B). (b) Topography of a compact slab made of calcium carbonate and kaolin microparticles with an average diameter of $400 \mathrm{~nm}$. The rms roughness is $115 \mathrm{~nm}$ and the surface correlation length is 900 $\mathrm{nm}$. (c) Intensity distribution collected over the same area at $z$ $=5 \lambda$ away from the interface.

scanned. A more elaborated model can be developed, which could account for variations of the refractive index along a rough surface profile. In this case, the properties of the "ef- 
fective surface" of the source can be quite different as one expects the effective refractive index contrast to diminish faster when the surface roughness increases.

In conclusion, we have shown that coherence properties of light scattered by random media can be examined using optical near-field techniques. We found that the spatial coherence length of the field measured at several wavelengths away from the surface relates to the interface local slope standard deviation. This behavior is different from that at larger distances from the interface where the coherence properties are dominated by the finite size of the scattering media as described by the Van Cittert-Zernike theorem. The classical coherence theory, on the other hand, requires infinitely extended sources. In our case, the extent of the source is practically infinite and the coherence properties are determined by modifications in the intensity angular distribution $J(\theta)$.

The results of this study suggest a number of applications. The morphological details at the surface of a random medium affect both the angular distribution of the radiant intensity and the spatial coherence properties of the radiation emitted. Measurements of the angular distribution of intensity in the far field require large sample size. Because of the natural sizes of the VSM involved in situations of practical interest, far-field measurements may need special optical arrangements or they could simply be impossible. The nearfield scanning approach described here can be applied to small-size samples. Because of the minute dimension of the probe and of the small scanning area, the sample can be considered to be a practically infinite source. Moreover, contrary to a near-field constant distance scanning operation, scanning in constant-height mode at micrometers away from the surface, permits a noncontact, noninvasive characterization of inhomogeneous interfaces. This NSOM collection mode can be considered as an intrinsically passive optical method for surface characterization, which may also be of practical use in some biological applications.

Our results demonstrate that second-order spatial coherence properties of the field in the close proximity of the sample surface can provide information about the statistical properties of the interface. They also suggest the possibility to adjust the coherence properties a few wavelengths away from the interface by modifying the statistical properties of the surface.

We are pleased to acknowledge useful discussions with Emil Wolf.
[1] W. H. Carter and E. Wolf, J. Opt. Soc. Am. 65, 1067 (1975).

[2] J. W. Goodman, Statistical Optics (Wiley, New York, 1985).

[3] S. Chandrasekhar, Radiative Transfer (Dover, New York,
1960).

[4] E. Wolf and W. H. Carter, Opt. Commun. 50, 131 (1984).

[5] A. Dogariu and G. D. Boreman, Opt. Lett. 21, 701 (1996). 